\title{
Recent Results from VERITAS
}

\author{
David Hanna (for the VERITAS Collaboration) ${ }^{* i}$ \\ Physics Department, McGill University, Montreal, Canada \\ E-mail: hanna@physics.mcgill.ca
}

\begin{abstract}
VERITAS (Very Energetic Radiation Imaging Telescope Array System) is an array of four 12metre atmospheric Cherenkov telescopes located near Tucson, Arizona, USA. It is sensitive to astrophysical gamma rays at energies above $100 \mathrm{GeV}$. Since becoming fully operational in September 2007, VERITAS has detected a variety of sources, including active galactic nuclei, pulsar wind nebulae, and supernova remnants. Gamma rays have also been observed from a starburst galaxy, a radio galaxy and an X-ray binary system. Searches for dark-matter annihilation and black-hole evaporation are a continuing part of the science program. This presentation will highlight recent results from VERITAS and outline plans for future upgrades and further observations.
\end{abstract}

35th International Conference of High Energy Physics - ICHEP2010,

July 22-28, 2010

Paris France

\footnotetext{
* Speaker.

${ }^{\dagger}$ see http://veritas.sao.arizona.edu/conferences/authors for a list of collaboration members
} 


\section{Introduction and Experimental Details}

$\mathrm{TeV}$ gamma-ray astronomy has strong connections to particle physics, the central theme of the ICHEP conference series, and VERITAS [1] is one of the new generation of $\mathrm{TeV}$ instruments pursuing an active program in this area. In this contribution I will highlight some recent results which are connected to the astronomy side of particle astrophysics, namely recent discoveries of new sources of $\mathrm{TeV}$ gamma rays. For more details please visit the VERITAS public web-site (http://veritas.sao.arizona.edu).

The VERITAS detector comprises an array of four imaging atmospheric Cherenkov telescopes (IACTs) located at an altitude of $1.3 \mathrm{~km}$ near Mount Hopkins in southern Arizona. Each telescope consists of a 12-metre reflector which collects Cherenkov light from extensive air showers and directs it onto a 'camera' made from 499 photomultiplier tubes read out by 500 MS/s FADCs. Each camera has a field of view $3.5^{\circ}$ in diameter. The array was built in stages between 2003 and 2007 with four-telescope observations beginning in September, 2007.

VERITAS covers the energy range from approximately $100 \mathrm{GeV}$ to beyond $30 \mathrm{TeV}$ with a resolution of between 15 and $20 \%$ and single-event angular resolution (68\% containment) of $0.1^{\circ}$. It can make a five-sigma detection of a source with a flux of $1 \%$ of that of the Crab Nebula in less than 30 hours. More details can be found in [2]. As of the summer of 2010, VERITAS had made 32 detections, of which 15 were discoveries; the rest were already known to be VHE emitters. Eighteen of the detected sources are extragalactic, seven are galactic and seven have not yet been identified with objects known at other wavelengths.

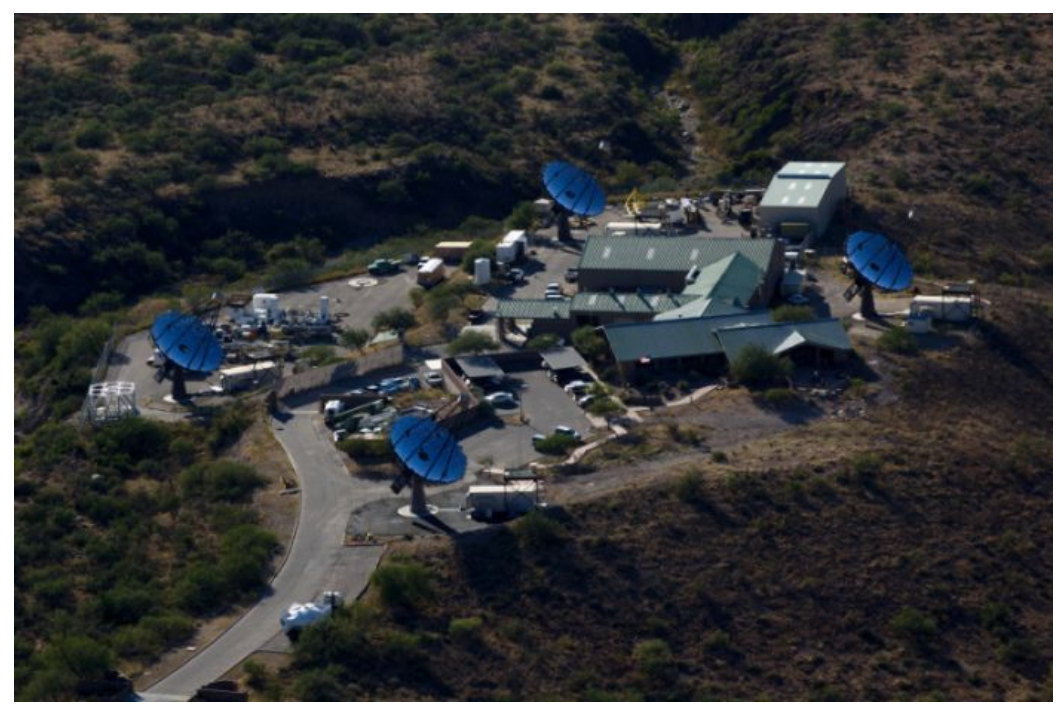

Figure 1: The VERITAS array at the Whipple Observatory base camp near Mount Hopkins in southern Arizona.(photo: S. Criswell SAO)

\section{Extragalactic Sources}

Most of the extragalactic sources studied by VERITAS are blazars, active galactic nuclei (AGN) with one jet pointed along our line of sight. The aim of these studies is to understand jet production by supermassive black holes and the physics behind gamma-ray production therein. 
Here multiwavelength studies can be useful in determining whether accelerated protons or electrons are ultimately the source of the gamma rays. Another goal is to measure the level of extragalactic background light (EBL) via its energy-dependent attenuation effects on VHE gamma rays.

PKS 1424+240: One the most recent AGNs to be detected by VERITAS is PKS 1424+240 [3]. This object is the first $\mathrm{TeV}$ source to be detected as the result of a trigger from the Fermi satellite [4], which has all-sky sensitivity at $\mathrm{GeV}$ energies. It became the subject of a multiwavelength campaign involving VERITAS, Fermi, Swift [5] and the MDM observatory. The energy spectrum derived from VERITAS data acquired between February 19 and June 21, 2009 is plotted in figure 2. The data can be fit with a steep (index $=3.8 \pm 0.5 \pm 0.3$ ) power law above $140 \mathrm{GeV}$. Contemporaneous Fermi observations in the range $0.1<E<300 \mathrm{GeV}$ yield a power-law spectrum with an index of $1.73 \pm 0.07 \pm 0.05$, indicating that there is spectral break between the two ranges. This is expected for distant emitters since high energy gamma rays are attenuated by pair-production reactions with infra-red photons (the EBL) along the way. Unfortunately, the redshift of PKS 1424+240 is unknown; this is a common feature of BL Lacs, the class of AGN to which this source belongs, since a defining characteristic is their lack of spectral lines. Assuming the increase in power-law index is due to the EBL effect and making use of recent EBL models, one can derive a redshift upper limit of $z<0.66(95 \% \mathrm{CL})$ making this possibly one of the most distant sources to be detected in $\mathrm{TeV}$ gamma rays. However spectral modeling can explain the break as intrinsic to the source and can accommodate more modest $z$ values. It is clear that progress here requires independent distance estimators.

1ES 1218+304: In contrast to the previous source, the AGN 1ES 1218+304 has a known redshift: $z=0.182$. It was previously detected by MAGIC [6] and VERITAS [7] with a steady flux at $\sim 6 \%$ of the Crab Nebula and no evidence for time variability. The power-law-spectrum index (approximately 3 ) from these observations presents a challenge; a source at such a redshift should have had its spectrum steepened considerably by the effects of the EBL. Unfolding the attenuation effects using models of the EBL results in hard intrinsic spectra (indices less than 1.3) [8] which are difficult to explain with the simplest models of blazar gamma-ray production. A model [9] that posits production over kiloparsec distance scales far out in the jet is able to accommodate the data however. VERITAS acquired new data [10] on this source between December, 2008 and May, 2009. During this time a flare was detected, as shown in the light curve displayed in figure 3 . This rapid time variation is evidence in favour of a much smaller emission region $(<0.01 p c$, assuming a Doppler factor of 20) and thus challenges theorists to come up with other mechanisms for producing hard blazar spectra.

M82: A final highlight of the VERITAS extragalactic program is the detection of $\mathrm{TeV}$ emission from the starburst galaxy M82, also known as the Cigar Galaxy. This is a spiral galaxy with a dense core where stars are being formed at a rate ten times that of the Milky Way. This leads to many supernova explosions as well as collisions of intense stellar winds, both thought to be responsible for producing most of the charged cosmic rays in a galaxy. Concomitant gamma-ray production is expected from $\pi^{0}$ s produced by cosmic ray collisions. VERITAS observed M82 for 140 hours over a two-year time period and detected gamma rays at energies greater than $700 \mathrm{GeV}$ [11] and a flux level of $0.9 \%$ of the Crab. This five-sigma post-trials detection is the first evidence for gamma-ray production in a starburst galaxy and provides support for the cosmic-ray origin paradigm. 


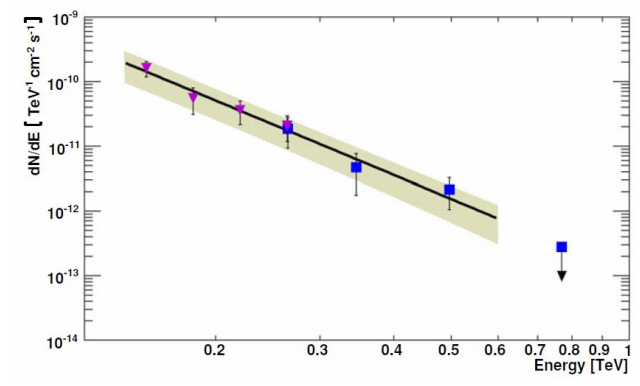

Figure 2: Time-averaged differential photon spectrum of PKS 1424+240. The triangles and squares correspond to analyses with different cut values and the line is a powerlaw fit explained in the text. The shaded region shows the systematic uncertainty of the fit, dominated by a $20 \%$ uncertainty on the energy scale.

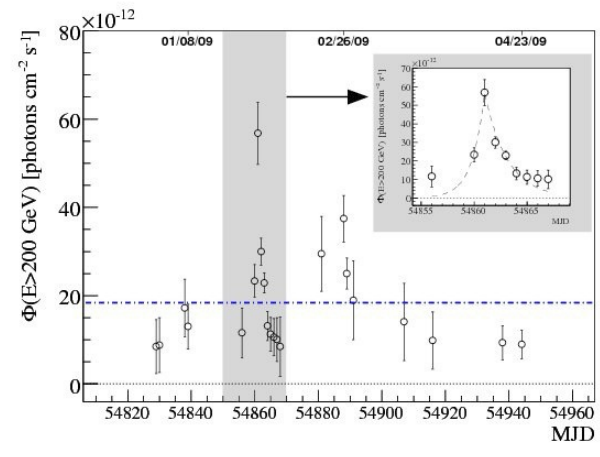

Figure 3: Light curve for 1ES 1218+304 between December 29, 2008 and April 23, 2009. Data points represent integral flux above $200 \mathrm{GeV}$ assuming a power-law spectrum with index 3.07. The flare portion (inset) is fit with two exponentials $\left(e^{\lambda t}\right)$ yielding $\lambda_{\text {rise }}=0.86$ day and $\lambda_{\text {fall }}=-0.47$ day.

\section{Galactic Sources}

VERITAS Galactic observations target supernova remnants and pulsar wind nebulae as well as binary systems. Candidates are selected by their similarity to known $\mathrm{TeV}$ sources and by their activity at other energies such as X-rays. A survey of the Cygnus region is also part of the program.

Cassiopeia A: This source is a young (330 years old) shell-type supernova remnant previously detected by HEGRA [12] and MAGIC [13]. VERITAS detected Cas-A using data from 22 hours of observations carried out in October and November 2007 [14]. The sky map is shown in figure 4 where it can be seen that the signal extent is approximately the same as the gamma-ray pointspread-function so with the data in hand we can only say that the source is consistent with being point-like. A power law with index of $2.6 \pm 0.2 \pm 0.2$ describes the data well over the range from 0.4 to $4 \mathrm{TeV}$. The integral flux above $1 \mathrm{TeV}$ is approximately 3.5\% that of the Crab. Modeling of the production mechanism for gamma rays by the Fermi-LAT team [15] using Fermi and VERITAS measurements slightly favours a hadronic origin over leptonic but either can be made to work.

Tycho's SNR: The remnant of the supernova observed by Tycho Brahe in 1572 was observed by VERITAS for 67 hours in 2008 and 2010 resulting in a five-sigma (post trials) detection of this source, the first time it has been detected in $\mathrm{TeV}$ gamma rays. Although $\mathrm{X}$-ray data from this object imply the existence of electrons accelerated to energies above $10 \mathrm{TeV}$, it has never been detected in gamma rays, even at $\mathrm{GeV}$ energies with EGRET and Fermi. The flux level is approximately $0.9 \%$ of the Crab above $1 \mathrm{TeV}$. Its spectrum between 1 and $10 \mathrm{TeV}$ can be described by a power law with index $2.0 \pm 0.5 \pm 0.3$. More details will be available in an imminent publication.

\section{VERITAS Upgrade}

VERITAS is undergoing an upgrade. During the summer of 2009 one of the telescopes was 


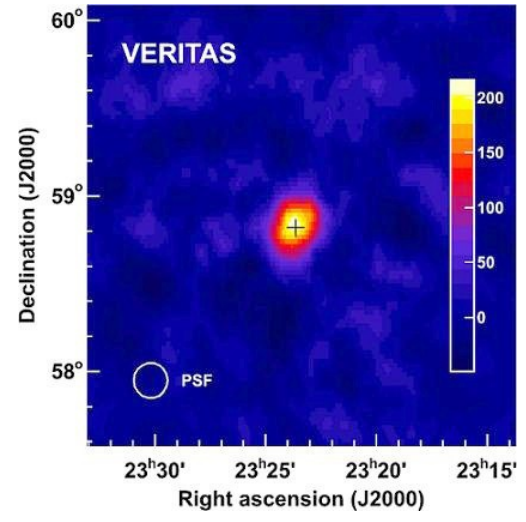

Figure 4: Smoothed sky map of excess counts for the Cas-A data set. The black cross indicates the measured position of the $\mathrm{TeV}$ emitter and the white circle the VERITAS point-spread-function.

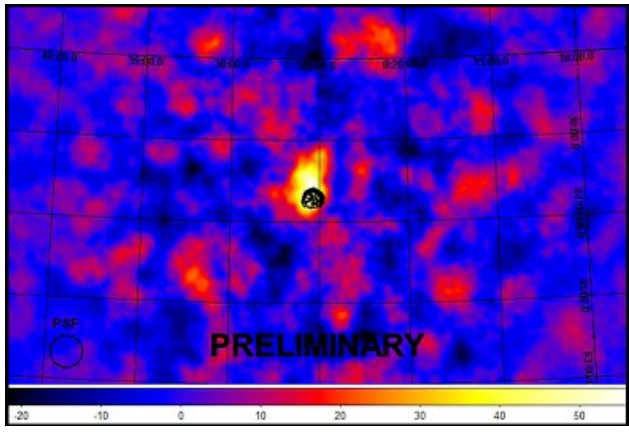

Figure 5: Preliminary sky map of excess counts from the Tycho data set. The black contours on the gamma-ray signal region are from Chandra ACIS measurements [16].

moved in order to make a more symmetric array with increased baselines, resulting in significantly improved sensitivity. Building on this, we plan to replace all phototubes with new ones having 50\% higher quantum efficiency, thereby increasing the Cherenkov photon collection efficiency by the same factor. A new FPGA-based camera trigger is an integral part of the upgrade, enabling a new array trigger with the potential to reduce background at the trigger level. The enhanced instrument will allow deeper, more sensitive observations and help VERITAS to remain a key player in this exciting field.

\section{Acknowledgements}

VERITAS research is supported by grants from the US Department of Energy, the US National Science Foundation, and the Smithsonian Institution, by NSERC in Canada, by Science Foundation Ireland (SFI10/RFP/AST2748), and by STFC in the UK. The author would like to thank the ICHEP2010 conference organizers for an interesting and enjoyable meeting.

\section{References}

[1] T. C. Weekes et al., Astropart. Phys. 17, 221 (2002)

[2] J. Holder et al., Astropart. Phys. 25, 391 (2006)

[3] V. Acciari et al., Ap.J 708, 100 (2010)

[4] A.A. Abdo et al., Ap.JS 183, 46 (2009)

[5] N. Gehrels et al., Ap. J 611, 1005 (2004)

[6] J. Albert et al. Ap. J 642, L119 (2006)
[7] V. Acciari et al., Ap.J 695, 1370 (2009)

[8] F Krennrich et al., Ap.J 689, L23 (2008)

[9] M. Boettcher et al., Ap.J 679, L9 (2008)

[10] V. Acciari et al., Ap.J 709, L163 (2010)

[11] V. Acciari et al., Nature 472770 (2009)

[12] A. Aharonian et al., A\&A 112307 (2001)

[13] A. Albert et al., A\&A 474937 (2007)

[14] V. Acciari et al., Ap.J 714, 163 (2010)

[15] A.A. Abdo et al., Ap.J 710, L92 (2010)

[16] J.S.Warren et al., Ap.J 634, 376 (2005) 